\title{
The Design of Smart Power Meter Based on ARM9 Microprocessor
}

\author{
Yujie Shi ${ }^{1, a}$ Xinhui $\mathrm{Du}^{2, \mathrm{~b}}$ \\ University of Technology,Shanxi, Taiyuan, China \\ University of Technology, Shanxi,Taiyuan, China \\ sy-jie@163.combduxinhui211@163.com
}

\begin{abstract}
.
Aiming at the disadvantages accuracy, great loss and unstableness by electronic power meter,the smart meter system based on ARM9 microprocessor was designed. Hardware circuit mainly includes the power acquisition module, data storage module, power module and two-way communication module. Electric meter software using the simplified Linux2.6.30 version of embedded system,meanwhile, procedure code written for various modules driver and application in Linux platform. Smart meter control panel using Qt/Embedded graphic development tools shows the user information on current electricity consumption and electricity rates.Experiment proved that the smart meter with high reliability solved the existing defects of electronic watt-hour meter , and had wide application.
\end{abstract}

Keywords: power meter;ARM9;embedded system;two-way communication

\section{Introduction}

Watt-hour meter is the important measure tool for economic accounting and energy saving management in power generation, transmission, distribution and utilization. It is improving with the continuous development of modern electronic technology, instrument technology. With the development of technology, and the demand of the market, the watt-hour meters with high precision, multi-function, low power consumption, are widely used in electric measurement. ${ }^{[1]}$

The diversity of function demands determines the diversity of the meters. They can be divided into active watt-hour meter, reactive watt-hour meter, multi rate 
time-sharing watt-hour meter, prepaid watt-hour meter, loss watt-hour meter, multi-function watt-hour meter and smart meter, etc. Multi rate time-sharing watt-hour meter appears after the peak\&valley price policy, meeting a great demand. It is designed to guide the user perform time-sharing, making reasonable and effective utilization of the electricity resources. It calculates electricity bill in segmentation, through separately metering electric energy at different times. The smart meter in this paper, has integrated function. It can not only achieve the time-sharing and provide users with customized services, but also evaluate the energy consumption of each household electrical appliances. ${ }^{[2]}$ Through the software, the saved data can be processed into statement for the single electrical appliance, generating visual graph, providing reference for the user to formulate reasonable energy-saving scheme. Such a powerful smart meter with reasonable price can improve residents' energy-saving consciousness, solve the problem of electric power shortage. It has great value on research and practice, and positive significance to promote social development. ${ }^{[3]}$

\section{System overview}

Structure design of overall system. The smart meter in this paper, is mainly used for residents indoor. It has time-sharing metering function, to adapt to the current time-sharing price policy in the domestic regions. First, it realizes the collection and storage of the electric energy data. Then, it adds time memory, to determine the division of time, providing precondition for time-sharing metering. In addition, smart meter has communication interface, for the two-way data communication between the user and Power Grid Corp collecting system. The smart meter is mainly composed of electricity detection module, external power supply module, S3C2440 ARM9 core processor, LCD display module, memory module and other peripheral circuit. The electricity detection module takes special energy metering chip ADE7758 as the core, to achieve the data such as active power, reactive power, voltage, current and frequency. Main control part is samsung S3C2440 processor with appropriate peripheral module circuit. The data obtained are 
processed through the processor and displayed on the LCD screen, for users to view at any time. The communication module uses Ethernet to realize two-way communication between the smart meter and the Power Grid Corp collecting system. $^{[4]}$

Operation mechanism of system. Three-phase load circuit includes phase A, phase $\mathrm{B}$, phase $\mathrm{C}$ and neutral wire $\mathrm{N}$. When starting the load, the current and voltage via the lines are the electrical parameters in the meter. The collected voltage, current signals via attenuation filter circuit to metering chip ADE7758. When the metering chip port receive the corresponding signal, after the internal filtering, differential and integral operations, it saves all kinds of parameters in the corresponding register. The metering of power in the whole system is mainly performed by the CPU. It reads data from the corresponding register in the metering chip ADE7758, in fixed time, then obtains the value through calculation. The communication between the main controller and the metering chip is performed through the SPI interface. In case of abnormal state (power off), ADE7758 will perform interruption on MCU through the interrupt request IRQ.

\section{Circuit design of main module}

Design of acquisition circuit. The metering module is chip ADE7758 with appropriate peripheral circuit. The value range of the input electrical parameter allowed by the chip is limited, so attenuation filtering on the voltage signal and current signal collected from the three-phase load circuit is required. The sampling of voltage signal and current signal are completed by the transformer. The sampling circuit in A phase is shown in Fig. 1. R4, R5, R11, R16 are the anti aliasing filter networks for each port, and each input port has four groups of inversion diodes, to constitute protection circuit of each line. The sampling circuit design of Phase $\mathrm{B}$ and $\mathrm{C}$ is the same with the phase $\mathrm{A}$. The input signal of IAP and IAN channels should be voltage signal, so the resistor R1 get voltage directly from the secondary side of the current transformer. The value R1 get is related to the 
output value of current transformer secondary side. ${ }^{[5]}$ The acquisition circuit is shown in the Fig. 1.

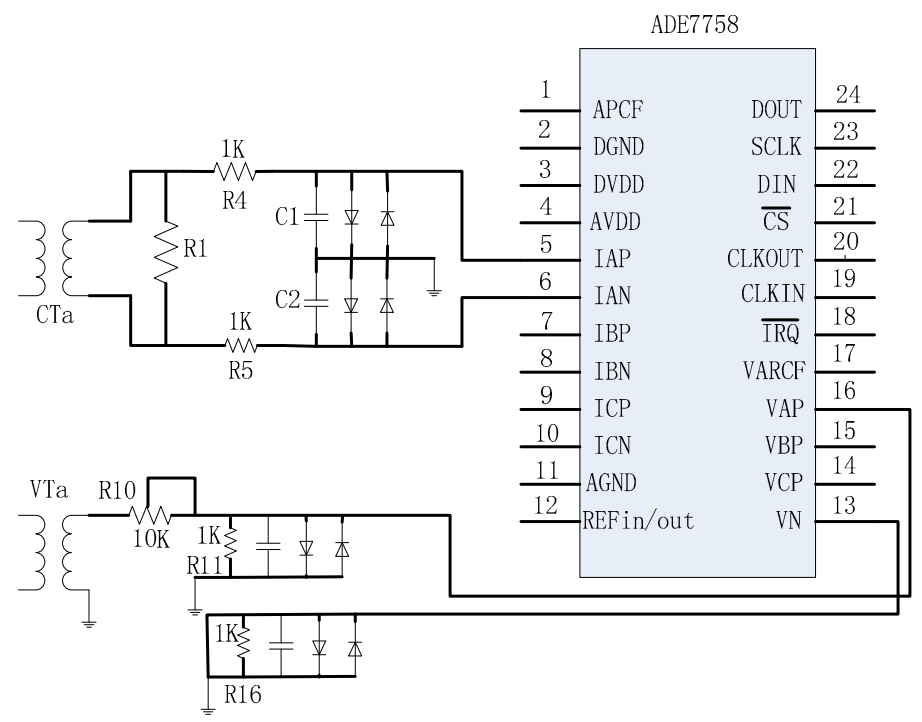

Fig. 1 The acquisition circuit

Interface design of ADE7758 and MCU. In sampling circuit, the transformer can not only transform grade, but also perform electrical isolation. It separates the high voltage on the primary side and weak voltage on the secondary side. In this case, it can connect ADE7758 SPI interface (DOUT, SCLK, DIN, CS) with MCU, through the $1 \mathrm{k} \Omega$ resistance. The APCF and VARCF pulse interfaces of ADE7758, need the optoelectronic isolation unit TLP421 to realize anti-jamming. Optical coupling can realize the input and output electrical isolation, and oneway signal transmission. It has good insulation ability and anti-interference ability. And light coupling input end has low impedance device of current working model, making it strong common mode rejection capability.

Interface design of ADE7758 and MCU. In sampling circuit, the transformer can not only transform grade, but also perform electrical isolation. It separates the high voltage on the primary side and weak voltage on the secondary side. In this case, it can connect ADE7758 SPI interface (DOUT, SCLK, DIN, CS) with MCU, through the $1 \mathrm{k} \Omega$ resistance. The APCF and VARCF pulse interfaces of ADE7758, need the optoelectric isolation unit TLP421 to realize anti-jamming. 
Optical coupling can realize the input and output electrical isolation, and oneway signal transmission. It has good insulation ability and anti-interference ability. And light coupling input end has low impedance device of current working model, making it has strong common mode rejection capability.

Design of storage module. The storage resources on chip in the embedded system is limited, which can't meet the requirements of system development. So to establish an efficient storage system is the basic work in embedded system development. At present, NOR Flash memory is expensive, and the volume is small. So NAND Flash+SDRAM plan is acceptable, as shown in the figure. Users can start and guide the system from the NAND Flash, and perform the main program in the SDRAM. S3C2440 meet the requirements of this plan, because it has an internal SRAM buffer (Steppingstone). When starting the system, the first $4 \mathrm{~kb}$ byte code in the NAND Flash memory will be automatically loaded into the Steppingstone, and automatically execute the code, starting the system in the NAND Flash. In order to manage the requirements of storage on speed and data width, S3C2440 uses storage controller to provide necessary storage control signal for the access and management of memory not on the chip. ${ }^{[6]}$

Design of communication interface. The existing peak\&valley timesharing meter, prepaid watt-hour meter and the watt-hour meter with infrared meter reading and long-distance meter reading function, can not meet the requirements of the real-time interaction in the smart grid. They are not real smart meters. Under the smart grid architecture, the smart meter should have the metering function in real time, to provide power information with timing mark. It should not only provide useful information in real time for the high efficiency and energy saving management in power grid, but also have the function of two-way communication, which is the foundation of interaction.Two-way communication is required for smart meter. But S3C2440 has no Ethernet interface. In order to make S3C2440 possess the function of Ethernet, it is necessary to expand the network card interface. This system uses DM9000. This chip can be directly connected to the ISA bus, and it can also be connected with S3C2440. DM9000 has only two ports: 
address and data. The address port is used to enter the address of internal register. The data port is used to read and write in a register. CMD pin is used to differentiate between the two ports. When the pin is 0 , DM9000 transmission is for the register address. When the pin is 1 , the transmission is to read and write data. SA8, SA9 are connected to high level, SA4 - SA7 are connected to low level. AEN is connected to nGCS4 pin. Then, the DM port base address: 0x20000300, CMD pin is connected to the ADDR2 pin, to define the addresses of DM9000 ports, address port: 0x20000300 and data port: 0x20000304.

\section{Design of system software}

Embedded Operating System (EOS) is the control center of software and hardware resources. ${ }^{[7]}$ EOS is responsible for the allocation and scheduling of all the software and hardware resource in system. It controls and coordinates concurrent activities. This system takes the embedded Linux as operating system. The source code of Linux is open, and follows the GPL rules. The system selects Linux 2.6.30 version, and perfroms system trimming to fit for the embedded applications in this system. At the same time, in the Linux platform, it writes drivers and applications for each module. Meter control panel uses Qt/Embedded graphic development tools. The panel displays the current electricity consumption and electricity rates. Electricity metering module sends electricity information through the SPI interface to S3C2440. After data processing, the final information will be displayed on the LCD screen. ${ }^{[8]}$ When the system detects abnormal condition, the control panel displays alarm signals and possible causes. The whole system software flow diagram is shown in the Fig. 2. 


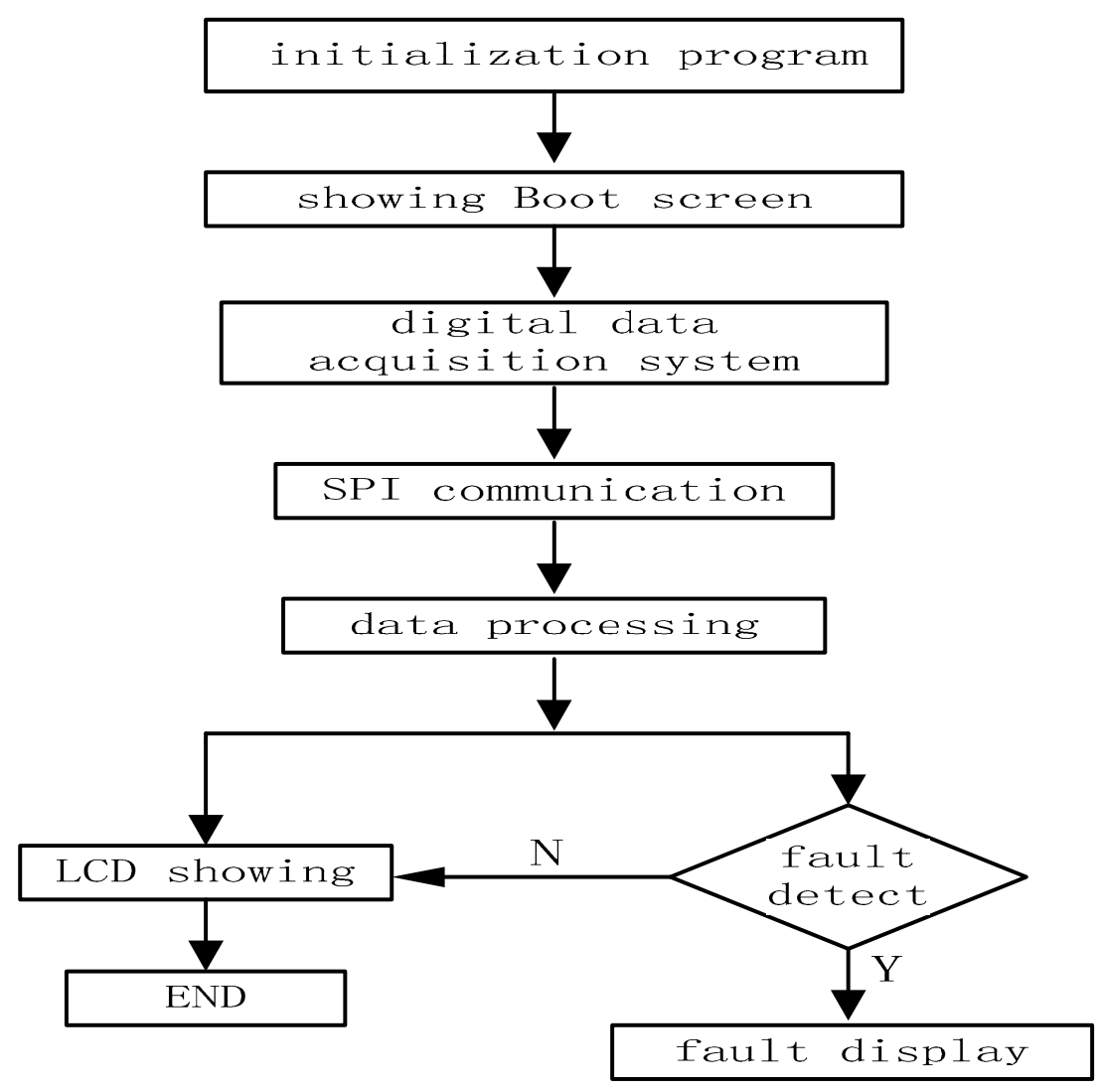

Fig. 2 whole system software flow diagram

\section{Conclusion}

This article introduces in detail the design scheme of the smart meter based on ARM9 microprocessor. Along with the gradually completed power grid reconstruction and the smart grid construction, the power terminal intelligence becomes the trend. The demand for smart meter will also get increased significantly. Smart meter system based on embedded technology has high reliability, less error, strong controllability, fast data communication, lower cost. It will get wide promotion and application.

\section{References}


[1]Xinhui Du,Qian Wang,The Design of Multi-Functional Power Meter Based on ADE7758 ,Electrical Engineering[J] 2012.2:42-44(in Chinese)

[2] Yixin $\mathrm{Yu}$, Wenpeng Luan.Smart grid and its implementation[J].Proceedings of the CSEE,2009,29(34): 1-8(in Chinese).

[3] Kai Zhang,Xinhui Du,The Research of Smart Meter Two-Way CommunicationBased on Advanced Metering Infrastructure,2012(in Chinese)

[4] Bobo Wang,Zheng Wang,ARM9 learning manual[M],2011(in Chinese)

[5] AD [Analog Devices].ADE7758:Polyphase Multifunction Energy Metering IC with per Phase Active and Reactive Powers[Z]. http://www.analog.com/, 2010.

[6] V.Vapnik,AnOverview of Statistical Learning Theory [J]. IEEE Transactions on Neural Networks, 1999, 10 (5): 988- 999

[7] Zongquan Deng,Xiangdong Jiang,Design of automotive intellectual control meter system based on embedded Linux system[J],microcomputer \& its applications,2014(in Chinese)

[8] Liang Chen,Fangcheng Lv,Research of Real-time Energy Measurement and Bidirectional communication Smart Meter, Proceedings of the CSEE,2011(in Chinese) 